Instituto Internacional de Investigación y Desarrollo Tecnológico Educativo INDTEC, C.A.

DOI: https://doi.org/10.29394/scientific.issn.2542-2987.2016.1.2.17.295-315

OAI-PMH: http://www.indteca.com/ojs/index.php/Revista Scientific/oai

\title{
Orientaciones Teóricas para el Desarrollo de la Comprensión Lectora Mediante el Uso de Estrategias Metacognitivas
}

\author{
Autora: Yelitza del Carmen Morillo Terán \\ Universidad Nacional Experimental "Rafael María Baralt", UNERMB \\ yecarmar72@hotmail.com \\ Zulia, Venezuela
}

\section{Resumen}

La investigación, tiene como propósito generar orientaciones teóricas para el desarrollo de la comprensión lectora mediante el uso de estrategias metacognitivas en el IUTEMBI Instituto Universitario de Tecnología "Mario Briceño Iragorry", ubicado en Valera, estado Trujillo. Las teorías referenciales se sustentarán en Pujol (2010), Pinzás (2013), entre otros. Para su desarrollo se asumirá el enfoque cualitativo a través del método fenomenológico sustentado en la hermenéutica que permitirá la comprensión de los fenómenos en sus variadas manifestaciones. Los informantes claves se seleccionarán siguiendo criterios propios de la investigación cualitativa, partiendo del hecho que son profesores de la institución antes mencionada y se comprometieron a participar voluntariamente en la investigación. De tal manera se considerarán como informantes tres docentes y tres estudiantes de las diferentes carreras que oferta esa casa de estudios. Para el desarrollo del estudio se utilizarán como instrumentos una entrevista no estructurada, grabaciones de audio, que permitirán estudiar en profundidad las unidades de análisis. Los hallazgos permitirán generar las orientaciones teóricas antes mencionada.

Palabras clave: comprensión lectora; estrategias metacognitivas; educación universitaria. 


\title{
Theoretical Guidelines for the Development of Reading Comprehension Using Metacognitive Strategies
}

\begin{abstract}
The research aims to generate theoretical development of reading comprehension guidance by using metacognitive strategies in the IUTEMBI University Institute of Technology "Mario Briceño Iragorry" located in Valera, Trujillo state. Referential theories will be based on Pujol (2010), Pinzás (2013), among others. For the qualitative development through the phenomenological method supported by hermeneutics that enable understanding of the phenomena in its various manifestations approach is assumed. Key informants will be selected according to criteria of qualitative research, based on the fact that teachers are the aforementioned institution and pledged to voluntarily participate in the research. So they are regarded as informers three teachers and three students of different races that offer this university. For the development of the study will be used as instruments an unstructured interview, audio recordings, that will study in depth analysis units. The findings allow the aforementioned theoretical generating orientations.
\end{abstract}

Keywords: reading; metacognitive strategies; college education.

Date Received: 15-08-2016

Date Acceptance: 30-08-2016 


\section{Introducción}

En la educación universitaria, comprender textos escritos es obligatorio para alcanzar las diferentes disciplinas; escritos es un requerimiento necesario durante el proceso de enseñanza aprendizaje, sin embargo, algunos estudiantes que ingresan a las universidades venezolanas, de manera constante, muestran debilidades en la comprensión lectora debido posiblemente al escaso manejo de estrategias metacognitivas por parte del docente que los lleven a superar este inconveniente.

Dado que, en la actualidad, la velocidad con la cual se elaboran y circulan las informaciones, es un reto para los estudiantes en todos los niveles el manejo de competencias para seleccionar información, resolver problemas y tomar decisiones que faciliten un alto rendimiento cognitivo. En virtud de ello, se hace obligatorio el dominio de la comprensión de textos, de acuerdo a los objetivos planteados, las características de la tarea en juego, así como también utilizar mecanismos de autorregulación en los procesos cognitivos. Sin embargo, leer más que un simple acto mecánico de descifrado de signos gráficos, es por encima de todo un acto de razonamiento, ya que de lo que se trata es de saber guiar una serie de argumentos hacia la construcción de una interpretación del mensaje escrito a partir de la información proporcionada por el texto y los conocimientos del lector, pero, a la vez, iniciar otra sucesión de testimonios para controlar el progreso de esa interpretación de tal forma que se puedan detectar las posibles incomprensiones producidas durante la lectura.

Por esa razón, surge mi interés por este estudio dirigido a generar orientaciones teóricas para el desarrollo de la comprensión lectora mediante el uso de estrategias metacognitivas en el IUTEMBI ubicado en Valera, estado Trujillo, el cual es de tipo cualitativo con enfoque fenomenológico sustentándose en el análisis y la interpretación de los testimonios aportados por los informantes claves, a los cuales se sumará un proceso de reflexión, 
que estará enmarcado en la creación de categorías emergentes que permitirán el basamento de aportes teóricos, dentro de la investigación.

\section{Teoría y Conceptos}

\subsection{Aproximación al objeto de estudio}

La comprensión lectora en la formación académica de los estudiantes universitarios se considera como uno de los pilares fundamentales para la adquisición del conocimiento en sus procesos formativos. En este nivel, señalan Vega y otros (2013), uno de los principales retos de los educandos es aprender los contenidos disciplinares mediante la resolución de tareas basadas en la comprensión e integración de información proveniente de múltiples documentos complejos.

En ese sentido, la comprensión de múltiples documentos constituye una de las principales fuentes del aprendizaje disciplinar. Por tanto, realizar este tipo de lecturas eminentemente intertextuales, para resolver tareas académicas o profesionales supone que el estudiante desarrolle habilidades para localizar, evaluar, utilizar las diversas fuentes de información a fin de construir e integrar significados coherentes sobre un tema o dominio en específico. Por ello, podemos asumir que la alfabetización para la lectura va más allá de adquirir la habilidad de leer, implica también contar con las de pensamiento crítico, toma de decisiones, resolución de problemas.

A este conjunto de habilidades, Rouet (2012), denomina alfabetización de múltiples documentos complejos, considerando la perspectiva teórica de la comprensión del texto y el discurso, para referirse a la construcción de significados a través de libros de texto, reportes científicos, periódicos, páginas web, entre otros, los cuales representan un desafío para ser comprendidos de forma integrada.

Este desafío, señala el autor, se manifiesta principalmente en dos aspectos: a) debido a que los textos no han sido creados para comprenderse 
de manera conjunta, el lector debe inferir las relaciones intertextuales a través de las diferentes fuentes de información; b) evaluar de forma crítica la fiabilidad y relevancia de la misma. A partir de lo anterior, los autores Ponce y Carrasco (2010), centraron sus investigaciones, realizadas en México, en dilucidar los procesos cognitivos y las habilidades que caracterizan la lectura competente a partir de múltiples documentos, pues los estudiantes de los distintos niveles educativos tienen dificultades para resolver tareas donde se requiera aprender con/a partir de varios textos.

Esto se debe a que los textos académicos a los cuales acuden los estudiantes en busca de información no siempre resultan fácilmente comprensibles. Al respecto, señalan los autores mencionados, se observan dificultades importantes en el nivel pragmático revelando interferencias en la construcción de sentido del texto, a veces por limitaciones de la competencia de los lectores, otras por problemas en la organización retórica de los mismos.

Dentro de ese contexto, Calderón y Quijano (2010) explican que suelen observarse aún en los estudios de tercer nivel, problemas inherentes a una pluralidad de dominios como: información general inadecuada, concepciones erróneas, tratamiento insuficiente de textos complejos, desconocimiento de términos generales y específicos. El primer dominio, señalan los autores, es decir la información inadecuada obstaculiza la construcción de esquemas mentales que permitan comprender el texto. Al respecto, Carlino (2012:71) justifica la dificultad de los estudiantes puntualizando, por una parte, que los textos académicos (científicos, destinados al profesorado) descuentan conocimientos que ellos no poseen. Por otra parte:

...dado que carecen de conocimientos específicos sobre los textos y carecen de las categorías de pensamiento de la disciplina en la que se han empezado a formar, los alumnos no saben que buscar en la bibliografía y se pierden en la maraña de información que contiene, sin lograr distinguir lo que es central para la materia de lo que resulta accesorio. 
Podemos afirmar que al ingreso a la universidad no está garantizada la competencia en lengua escrita, la cual permita enfrentar con posibilidades de éxito el desafío de los estudios universitarios en donde se incluye, entre otras cosas, el tratamiento de textos académicos.

Los problemas detectados, según Olarte (2013), están asociados a falta de información, en algunos casos, y en otros a la dificultad para el uso de estrategias de aprendizaje cognitivas/metacognitivas requeridas para la comprensión de los textos leídos. Al respecto, la autora señala que, en Venezuela, es un hecho lamentable, aceptado comúnmente, el fracaso de la escuela en lo referente a proveer a los estudiantes de educación primaria, así como siguientes niveles de habilidades para alcanzar la comprensión lectora. En consecuencia, el bajo nivel de comprensión lectora de los estudiantes de todo el sistema educativo venezolano, de los cuales no escapan los educandos del Instituto Universitario de Tecnología Mario Briceño Iragorri (IUTEMBI), es una de las principales problemáticas que enfrentan los profesores del país, y específicamente, el estado Trujillo.

Cabe señalar que esa realidad la evidencié en visitas reiteradas a las aulas, donde observé la existencia de una gran mayoría de estudiantes de ese instituto con debilidades para aplicar estrategias o técnicas eficaces para la comprensión lectora, enfrentando entre otros problemas, la carencia de un conocimiento acerca de cómo evaluar la fiabilidad y relevancia de la información de las diversas fuentes. Al consultar a profesores de diferentes disciplinas acerca de la comprensión lectora de sus estudiantes manifestaron que no se evidencia por parte de ellos el paso por los distintos niveles de adquisición, retención, integración, recuperación y transferencia de información, además, el nivel de legibilidad alcanzado no permite la comprensión del texto, encontrándose en un nivel de comprensión literal, es decir, poco trascienden el texto, su léxico es muy reducido, escasamente comprenden las normas gramaticales. Asimismo, en conversaciones con 
estudiantes de las diversas carreras que imparte el IUTEMBI, éstos me manifestaron que no poseen hábitos de lectura, pues aun cuando viven y conviven en ambientes de lectura los textos son vistos de manera utilitaria más no desde su praxis profesional. Por esa razón, surge mi preocupación por realizar este estudio, de carácter interpretativo, el cual me permita generar orientaciones teóricas para el desarrollo de la comprensión lectora mediante el uso de estrategias metacognitivas en el IUTEMBI ubicado en Valera, estado Trujillo, interpretando las opiniones de los actores sociales sobre el tema. De las consideraciones antes mencionadas, surgen las siguientes interrogantes:

¿Cómo perciben los actores sociales del IUTEMBI ubicado en Valera, estado Trujillo la comprensión lectora?

¿Cuáles son las estrategias metacognitivas utilizadas por los profesores del IUTEMBI Valera para desarrollar la comprensión lectora en los estudiantes?

¿Cuáles son las principales dificultades que enfrentan los estudiantes del IUTEMBI Valera para desarrollar la comprensión lectora?

\subsection{Propósito general de la investigación.}

Generar orientaciones teóricas para el desarrollo de la comprensión lectora mediante el uso de las funciones informativas en el IUTEMBI ubicado en Valera, estado Trujillo.

\section{Contexto Referencial}

\subsection{Comprensión lectora}

La comprensión de la lectura se concibe como un proceso mediante el cual el lector construye un significado en su interacción con el texto y que se logra alcanzar durante ese proceso se origina de sus conocimientos previos acumulados. En ese sentido, Parodi (2009:39), señala: 
Definimos la comprensión de un texto como un proceso que se plasma en una representación mental construida progresivamente sobre la base de inferencias automáticas y fundamentales para establecer la coherencia de base y se continúa reelaborando como proceso de aprendizaje a partir del texto y de los conocimientos previos a través del cual se generan múltiples procesos inferenciales y se construyen conocimientos diversos de tipo relacional.

Podemos afirmar que esta perspectiva teórica considera la comprensión textual como un proceso cognitivo complejo, el cual se materializa en una representación del discurso escrito, como resultado de mecanismos inferenciales, estrategias cognitivas y lingüísticas activadas por el lector de un modo estratégico en interacción tanto con el texto, el medio social, sus conocimientos previos. En ese sentido, Defior (2010:15) afirma: "la comprensión lectora es el producto de un proceso regulado por el lector, en el que se produce una interacción entre la información almacenada en su memoria y la proporcionada por el texto". Según Valero (2011) existen varios niveles de comprensión lectora: literal, inferencial, crítico que es el superior. El primero es el más básico, se refiere a la lectura en la cual se identifican el tema y los aspectos generales de la información, explicitados en el texto, en donde el estudiante se limita a extraer información más no interpretan su mensaje. Al respecto, Campos (2009.32) expresa la necesidad del estudiante las habilidades cognitivas necesarias para el alcanzar la comprensión lectora, definiéndolas como:

Las operaciones mentales que ejecutan los lectores al construir el sentido de un texto, las cuales se ponen en marcha desde antes de comenzar a leer porque se adaptan al propósito de lectura y al tipo de discurso narrativo, descriptivo, argumentativo, expositivo y conversacional.

En otras palabras, el estudiante al leer pone en marcha un conjunto de operaciones mentales, las cuales le permiten construir el significado global del 
texto, por tanto, cada estudiante ante una misma lectura y en idéntico contexto situacional, aporta interpretaciones diferentes o complementarias al mismo.

Lluvias de ideas: ante la situación, se pretende conseguir ideas creativas para resolver problemas o situaciones, antes que sean suministradas o cuando, a través de analogías, se trata de describir un tema, tópico, aspecto u objeto del contenido que se desarrolla. Pueden generarse mediante mapas mentales donde participa la totalidad del cerebro en la construcción de asociaciones libres y conceptos organizados a través de dimensiones visuales. Este tipo de mapas, señala Anderson (2010:212) son utilizados básicamente para motivar la creatividad conceptual en los estudiantes, siendo altamente idiosincráticos, por lo mismo, centrados en aprendizaje independiente, permitiendo la activación de procesos cognitivos como la inducción, deducción, clasificación, comparación, entre otros. Ahora bien, lo que hace diferente al mapa mental es que posibilita a los estudiantes representar las ideas. Mapas conceptuales: según Ontoria (2010:87) son estructuras jerarquizadas por diferentes niveles de generalidad o inclusividad conceptual, formados por conceptos, proposiciones y palabras de enlace. Son procedimientos gráficos que el estudiante puede utilizar para explicitar su conocimiento sobre conceptos y relaciones entre ellos en forma de proposiciones verbales. Al respecto, Campos (2011:129) señala que un mapa conceptual tiene por objeto presentar las relaciones significativas, entre conceptos utilizando para ello, proposiciones verbales.

\subsection{Dificultades para el desarrollo de la comprensión lectora}

Leer un texto no significa que se comprende, puede ocurrir que se presenten algunas dificultades para lograr la comprensión total de lo que se lee. Al respecto, Condori (2012:95), explica que se encuentran una serie de dificultades al momento de leer y comprender lo leído, las principales son: 
a) Dificultades para penetrar en el texto, en tanto que unidad de significados relacionales. b) dificultades para interactuar con la propuesta de organización textual realizada por el autor del texto. c) dificultades para identificar las ideas más pertinentes que globalizan la información del texto.

Dentro de ese contexto, Pinzas (2013:84), señala algunos de los errores más comunes en la enseñanza de lectura que atañen a la comprensión, esto basado en que nuestro sistema escolar tiene tres características específicas en el tema de la comprensión lectora: El uso extendido de las modalidades de enseñanza que enfatizan el aprendizaje memorístico, no facilitan entender, o ir más allá de la información recibida para utilizarla, desarrollando así estudiantes que no son mentalmente activos y no aplican sus conocimientos.

\subsection{Estrategias metacognitivas}

Las estrategias metodológicas son formas específicas de organizar nuestros recursos (tiempo, pensamientos, habilidades, sentimientos, acciones) para obtener resultados consistentes al realizar una tarea. En ese sentido, Suárez (2013:42) plantea que en la comprensión lectora se utilizan diversas estrategias, muchas de ellas en forma inconsciente, sin embargo, resulta interesante resaltarlas a nivel consciente si se desea mejorarlas. Entre ellas se encuentran:

- Identificar la idea central, las que la sustentan y las relaciones entre ellas.

- Fijar la atención conscientemente en todo el texto.

- Subrayar las ideas importantes.

- Recordar la secuencia de lo leído.

- Clasificar los propósitos de la lectura.

- Parafrasear la información.

- Formular preguntas a lo largo de la lectura sobre el contenido del texto, para determinar si los objetivos se están cumpliendo. 
- Elaborar y probar inferencias, interpretaciones, predicciones, hipótesis y predicciones.

- Resumir, sintetizar y extender el conocimiento obtenido mediante la lectura, representando algunas ideas en forma de imágenes visuales.

La metacognición implica, por un lado, la capacidad de los estudiantes para recordar y reproducir procedimientos, hacer tareas o resolver problemas, al mismo tiempo, ser conscientes de la coherencia de lo que están haciendo, evaluarlo para poder reorientarlo de ser necesario. En ese sentido, las estrategias metacognitivas: permiten tomar conciencia del proceso de comprensión y ser capaz de monitorearlo a través de la reflexión sobre los diferentes momentos de la comprensión lectora.

\section{Orientaciones Metodológicas}

\subsection{Naturaleza de la investigación}

Aquí, se presentan los fundamentos ontológicos, epistemológicos y metodológicos-técnico que guiarán el desarrollo de este estudio. En tal sentido, se conjugan los elementos necesarios para comprender la totalidad del fenómeno investigado en los contextos socio académicos en los que tiene lugar. De este modo, la orientación epistemológica de la investigadora se sustenta en la relación dinámica de interacción entre la ontología del fenómeno a estudiar, el método utilizado para estudiarlo y los fundamentos epistemológicos que aseguran la concreción de tal consideración. En correspondencia con lo planteado, el objeto a estudiar, por tratarse de un fenómeno humano se abordará sistemáticamente, entendiendo que sus partes están determinadas por el significante y significados que el todo y sus partes adquieren en el estos, en el cual se extiende la manifestación del objeto de estudio. 
De acuerdo con lo anterior, se asume la investigación como una indagación de carácter cualitativo, la cual según Sherman y Webb (1988), posibilite la comprensión de las diferentes facetas del aprendizaje en el contexto universitario, así como los significados que los actores sociales le asignan a la comprensión lectora mediante el uso de las funciones informativas. La investigación cualitativa tiene significados diferentes en cada momento. Una primera definición, aportada por Denzin y Lincoln (1994:2), destacan que ésta, "implica un enfoque interpretativo, naturalista hacia su objeto de estudio. Esto significa que los investigadores cualitativos estudian la realidad en su contexto natural, tal y como sucede, intentando sacar sentido de, o interpretar, los fenómenos de acuerdo con los significados que tienen para las personas implicadas.

Así como lo presenta Stake (1999), que sitúa las diferencias fundamentales entre la investigación cualitativa y la cuantitativa en tres aspectos fundamentales: a) La distinción entre la explicación y la comprensión como propósito del proceso de indagación. b) La distinción entre el papel personal e impersonal que puede adoptar el investigador. c) La distinción entre conocimiento descubierto y conocimiento construido.

Para Stake (1999), la primera característica diferenciadora de la investigación cualitativa no se asienta en el enfrentamiento entre dato cualitativo versus dato cuantitativo, sino que se sitúa en el terreno epistemológico. El objetivo de la investigación cualitativa es la comprensión, centrada en la indagación de los hechos; mientras que la investigación cuantitativa fundamentará su búsqueda en las causas, persiguiendo el control y la explicación. Desde la investigación cualitativa se pretende la comprensión de las complejas interrelaciones que se dan en la realidad.

La segunda característica considerada por este autor, destaca de la investigación cualitativa el papel personal que adopta el investigador desde el comienzo de la investigación, interpretando los sucesos y acontecimientos. 
En la investigación cualitativa lo que se espera es una "descripción densa", una "comprensión experiencial" y "múltiples realidades". Como tercera característica diferenciadora de la investigación cualitativa, Stake (1999), argumenta que en ésta el investigador no descubre, sino que construye el conocimiento. En correspondencia con estos planteamientos se asume la presente investigación como un estudio cualitativo, en el que se busca generar categorías de análisis que fundamenten el desarrollo de la comprensión lectora mediante el uso de las funciones informativas. En consonancia con su naturaleza cualitativa desde la perspectiva ontológica, la investigación propuesta busca considerar la realidad de los estudiantes universitarios como dinámica, global y construida en un proceso de interacción con la misma. En el plano epistemológico, la investigación asume un carácter inductivo, partiendo de la realidad de los profesores y estudiantes que actuarán como informantes y de los datos que éstos aporten para llegar a una categorización posterior. En un plano metodológico, se sitúan las cuestiones, referidas a las distintas vías o formas de investigación en torno a la realidad. Desde este nivel el diseño de la investigación tendrá un carácter emergente, construyéndose a medida que se avanza en el proceso de investigación, a través del cual se puedan recabar las distintas visiones y perspectivas de los informantes. Desde un nivel técnico, en el cual se incluyen las técnicas, instrumentos y estrategias de recogida de información, en esta investigación se utilizaron técnicas que permitirán recabar datos que informarán sobre la particularidad de las situaciones, permitiendo una descripción exhaustiva, densa de la realidad concreta objeto de investigación, en este caso, se hará uso de la entrevista en profundidad y de la observación participante.

Para lograr los propósitos planteados se desarrollará una investigación fenomenológica, a través de la cual se busca abordar los significados que tienen para los actores sociales la comprensión lectora buscando con este tipo de investigación explicitar la esencia de la experiencia de los actores en torno 
a esta temática. Cabe destacar que en los estudios fenomenológicos de acuerdo con Apps (1991: 133), se deben desarrollar seis fases: a) descripción del fenómeno, b) búsqueda de múltiples perspectivas, c) búsqueda de la esencia y la estructura, d) constitución de la significación, e) suspensión de enjuiciamiento, y f) interpretación del fenómeno.

En correspondencia con esto, se desarrollará el estudio en un marco sistémico estructural que permitirá develar el significado de la comprensión lectora mediante el uso de las funciones informativas. De acuerdo a ello, el fenómeno a estudiar se originará en una concepción teórica de la comprensión lectora en el contexto universitario, en el cual se enmarcan los actores sociales que operan tal conceptualización y que generan múltiples interrelaciones que sólo pueden ser comprendidas en el marco de su entorno.

\subsection{Tipo de investigación}

Esta investigación está enmarcada siguiendo un marco de trabajo que se fundamenta en la investigación cualitativa bajo el modelo fenomenológico hermenéutico, entendido como un método de investigación por el cual se centra la atención en una descripción densa y una comprensión experiencial de acción transformadora, según Goetz y Lecompte (2012:13-14), de "las características de las variables y fenómenos, con el fin de generar y perfeccionar categorías conceptuales, descubrir y validar asociaciones entre fenómenos o comparar los constructos y postulados generados a partir de fenómenos observados en escenarios distintos".

\subsection{Técnicas e instrumentos de recolección de datos}

En este contexto, se considera la investigación como un proceso en el que intervienen una serie de factores: materiales, financieros, metodológicos y fundamentalmente humanos, cada uno de ellos requiere ser optimizado en su uso, para cuyo efecto el requisito básico es conocerlos en todas sus 
características, potencialidades y limitaciones, sobre cuya base se realiza una acción científica. Y es aquí, donde la investigación cobra su real dimensión, constituyéndose en una estrategia viable para el conocimiento de toda esta complejidad, a partir del uso de medios que permiten obtener la información necesaria, precisa, pertinente al objeto de nuestro estudio. En ese sentido, el enfoque cualitativo utilizado, requiere el uso de técnicas interactivas para la recolección de datos. Para el caso específico de la investigación, las estrategias e instrumentos serán:

- La observación no participante estará destinada al registro de datos generales, como información referente al contexto histórico y sociocultural de la comunidad, incorporando características cuantitativas (de cobertura escolar, población docente, de alumnado, condiciones de infraestructura y mobiliario), y cualitativas (diferentes acontecimientos suscitados, como reuniones, asambleas, eventos de capacitación, entre otros)

- La entrevista, entendida como una comunicación entre la investigadora y el sujeto de estudio a fin de obtener respuestas a interrogantes planteadas de manera abierta, libre, sin limitaciones. La opción por esta modalidad obedece al hecho de generar respuestas argumentativas, explicativas respecto de las actitudes, concepciones, expectativas y comentarios sobre el desarrollo de la comprensión lectora mediante el uso de las, para lo que se utilizarán como medios las guías de entrevista semiestructurada, instrumento aplicado de acuerdo al tipo de informantes (profesores, estudiantes), con preguntas preferentemente abiertas.

- Revisión documental de documentos de planificación diversos, información y comunicación, registros de capacitación docente, materiales educativos, propuestas curriculares y otros referidos al funcionamiento del proceso de enseñanza en la institución universitaria seleccionada.

- Los datos obtenidos con estos instrumentos, de guías de observación y entrevistas, no darán cuenta de todo cuanto se vio en la realidad si no son 
complementados por un registro diario del trabajo de campo, el mismo que contiene información del contexto de las relaciones informales y situaciones cotidianas.

La elaboración de este registro tendrá dos momentos: el primero, la libreta de notas de campo, que contiene información abreviada acerca de los sucesos en la universidad y que será posible percibirlos (desde el momento en que se hace presente hasta el momento de abandonarla). Segundo, para no perder el detalle de esta primera información, inmediatamente como sea posible, se ampliarán estas anotaciones en un diario de campo, registrando de manera minuciosa y retrotrayendo a la memoria todo cuanto pase durante la observación realizada, además de las impresiones que surgirán durante el trabajo de campo. El utilizar las entrevistas permite conocer íntimamente a las personas, ver el mundo a través de sus ojos, e introducirse vicariamente en sus experiencias. La investigadora intentará construir una situación que se asemeje a aquellas en las que las personas hablan naturalmente entre sí sobre cosas importantes. El objetivo es que la entrevistadora logre saber el modo en que los informantes se ven a sí mismos y a su mundo, obteniendo información precisa de acontecimientos pasados y de actividades presentes. A efectos de la presente investigación se seguirá una entrevista semiestructurada en función de responder a los propósitos que orientan el estudio.

\subsection{Categorización}

Según Rodríguez (2011), la categorización, hace posible clasificar conceptualmente las unidades que son cubiertas por un mismo tópico. Las categorías soportan un significado o tipo de significado y pueden referirse a situaciones, contextos, actividades, acontecimientos, relaciones entre personas, comportamientos, opiniones, sentimientos, perspectivas sobre un problema, métodos, estrategias, procesos. Para analizar la realidad observada 
en esta investigación es necesario categorizar la información recopilada. Para ello, se toma como unidades generadoras las definidas en los propósitos del estudio y formuladas a través de las interrogantes que guíen la entrevista. Sin embargo, éstas pueden variar de acuerdo a la información recolectada, así como de la convergencia y divergencia suscitada entre los informantes. Las categorías así obtenidas son ordenadas, codificadas en función de su vinculación utilizando códigos para diferenciar en la transcripción los elementos que las definen y que las diferencian unas de otras. Para lograr categorizar la información recopilada de las entrevistas realizadas se considerará el uso de dos matrices en la primera de ellas, se considerarán cuatro columnas la primera destinada a precisar las categorías de análisis, en la segunda se señalarán las dimensiones correspondientes a cada categoría, en la tercera columna se transcribirá textualmente la información aportada por cada informante, en tal sentido, se utilizarán seis matrices, para reflejar la información que será aportada por los seis informantes considerados para el estudio.

\section{Conclusiones}

Según los propósitos de la investigación particularmente como investigadora, puede concluir de la manera siguiente:

Realicé el primer contacto con los profesores en sus propias aulas de clase, donde les explicaré brevemente los fines para los cuales se requería de entrevistas partiendo además, de la consideración inicial de las ideas que surgirán de mi propia experiencia, dadas las razones voluntariamente accedieron a formar parte del proceso en estudio, las cuales darán paso a un proceso de diálogo donde es considerada la entrevista en profundidad, por las características anteriormente descritas.

En ese sentido, una vez grabadas las entrevistas serán transcritas a través de un formato computarizado en cuadros que se dividirán en: número 
de línea, representando la línea discursiva del momento, unidades discursivas en referencia al contenido del discurso del entrevistado, y categorías donde se reflejará mi interpretación al momento de su análisis; todo esto en estilo de horizontalidad, bajo el modelo de Moustakas (1994) desde sus constructos de la fenomenología, los cuales serán descritos más adelante; es de este modo como quedarán conformados los cuadros de representación visual de las entrevistas.

Asimismo, para analizar los datos se utilizará el método de las comparaciones constantes propuestas por Strauss y Corbin (2002:25) el cual se caracteriza por la generación de teoría que emerge de los datos, definido como un "conjunto de categorías bien construidas, por ejemplo, temas y conceptos, interrelacionadas de manera sistemática por medio de oraciones que indican relaciones, para formar un marco teórico que explica algún fenómeno social, psicológico, educativo, de enfermería o de otra clase".

La teoría permite establece una nueva red de relaciones entre las partes constituyentes, construidas a partir de la organización de los hechos observados, analizados, representados conceptualmente, constituyendo el aporte específico de la investigación. Durante el proceso de análisis, se entrecruzarán los datos codificándolos de dos formas: abierta y axial, analizando e interpretando la información cualitativa a partir de las entrevistas en profundidad realizados durante el estudio.

El método de comparaciones constantes se utilizará a través de cuatro fases: comparaciones de fragmentos de entrevistas, integración de categorías y sus propiedades, delimitación construcción, escritura de la teoría. El análisis se realizará con base en la narrativa de los sujetos que, de acuerdo con Bruner (2006:53) hacen referencia a una forma de relato para describir acontecimientos de la vida humana: "el relato se imbrica con la vida de la cultura, e inclusive se vuelve parte integrante de ella" y, el autor añade, la 
fecundidad del lenguaje permite narrar distintas versiones fundamentales para las interacciones sociales.

\section{Referencias}

Anderson, R. (2010). "El proceso de la comprensión lectora". Editorial Aljibe. Madrid.

Apps, D. (1991). "Tradición y enfoques en la investigación cualitativa". Editorial Aljibe. Madrid.

Bruner, J. (2006). “La fábrica de historias. Derecho, literatura, vida”. Fondo de Cultura Económica. Buenos Aires.

Calderón, A. y Quijano, J. (2010). “Características de comprensión lectora en estudiantes universitarios". Revista Estudios Socio-Jurídicos, Vol. 12, № 1. Bogotá.

Campos, E. (2009). "Gramática textual y enseñanza de la lengua”. Editorial Santillana. Buenos Aires.

Carlino, P. (2012). "Escribir, leer y aprender en la universidad: una introducción a la alfabetización académica". Fondo de Cultura Académica. Buenos Aires.

Condori, L. (2012). "Aplicación de estrategias metacognitivas para mejorar la comprensión lectora". Ediciones UNED. Madrid.

Defior, S. (2010). "Las dificultades de aprendizaje: un aprendizaje cognitivo". Editorial Aljibe. Madrid.

Denzin, N. y Lincoln, Y. (2011). "Manual de investigación cualitativa". Editorial Morata. Madrid.

Goetz, J. y Lecompte, M. (2012). "Etnografía y diseño cualitativo en investigación". Editorial Morata. Madrid.

Moustakas, C. (1994). "Métodos de investigación fenomenológica". Editorial Eunsa. Madrid. 
Olarte, N. (2013). "El problema de la comprensión lectora”. Editorial Magisterio. Bogotá.

Ontoria, A. (2010). "Mapas conceptuales. Una técnica para aprender". Editorial Narcea. Madrid.

Parodi, G. (2009). “Comprensión de textos escritos”. Ediciones Edudeba. Buenos Aires.

Pinzás, J. (2013). "Metacognición y lectura”. Pontificia Universidad Católica del Perú. Lima, Perú.

Ponce, A. y Carrasco, F. (2010). "Habilidades para una lectura competente". Editorial Trillas. México.

Pujol (2010). "Ayudar a enseñar”. Editorial Aique. Buenos Aires.

Rodríguez, G. (2011). "Metodología de la investigación cualitativa". Ediciones Aljibe. Madrid.

Rouet, J. (2012). "Efectos de la información en la comprensión de textos instruccionales". Editorial UNED. Madrid.

Ruedas, M. y otros (2007). "Epistemología de la Investigación Cualitativa. Universidad Experimental Pedagógica Experimental". Maracay.

Sherman y Webb (1988). "Métodos de investigación en ciencias sociales". Editorial Planeta. Madrid.

Strauus, A. y Corbin, J. (2002). "Bases de la investigación cualitativa. Técnicas y procedimientos para desarrollar la teoría fundamentada". Ediciones Universidad de Antioquia. Colombia.

Stake, R. (1999). "Investigación como estudio de casos". Editorial Morata. Madrid.

Suárez, E. (2013). "Estrategias de comprensión lectora". Editorial Magisterio. Bogotá.

Valero, M. (2011). "Psicolingüística”. Editorial Narcea. Madrid. 


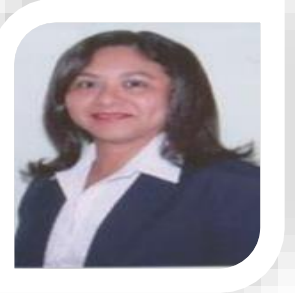

Nació en San Rafael de El Tigre, Municipio Baralt, Estado Zulia, el 17/09/1972. Estudiante de la Universidad Nacional Experimental "RAFAEL MARÍA BARALT" en el DOCTORADO EN EDUCACIÓN. Estudios de postgrado: Universidad "Valle de Momboy". Título obtenido: ESPECIALISTA EN EVALUACIÓN EDUCACIONAL. Estudio de Pregrado: U.N.E.R.M.B. Titulo Obtenido: LICENCIADA EN EDUCACIÓN, ÁREAS: Lenguaje y Ciencias Naturales. Ha participado en cursos, talleres y simposios sobre Análisis Institucional UNERMB. 1994. Implantación del Programa de Estudio de la Educación Religiosa Escolar I y II Etapa de la Educación Básica. Ministerio de Educación 1996. Fortaleciendo la Familia Diócesis de Cabimas. Sec. De Pastoral Social y Ministerio de la Familia. Duración 28 horas. Julio 1999. Capacitación y Actualización sobre el Nuevo Diseño Curricular, dirigido a los Docentes en servicio de la I Etapa de Educación Básica. Ministerio de Educación, Cultura y Deporte. Julio 2000. Operador en Computadora Registrado en el ministerio de Educación. Duración 48 horas. 2001 Importancia y efecto de la Droga en los niños, niñas y adolescentes. Ministerio de Educación, Cultura y Deporte. Enero 2003. Experiencia laboral: Escuela "Hilarión Alberto Viloria". Docente de Aula. Desde: 02-09-1995. Hasta 2013. Facilitadora de la Misión Sucre, Desde: 04-09- 2006 Hasta 07-2013. Actualmente Docente con Función Supervisora desde el 03/06/2013 hasta la presente.

El contenido de este manuscrito se difunde bajo una Licencia de Creative Commons ReconocimientoNoComercial-Compartirlgual 4.0 Internacional 